\title{
EVALUATION OF OCULAR PROSTHESIS FABRICATED BY RAPID 3D PRINTING TECHNOLOGY
}

\author{
Ahmed M. Hashem, ${ }^{1}$ Magdy M. Badawy, ${ }^{2}$ Mohamed A. Helal ${ }^{3}$
}

\begin{abstract}
Objective: was to compare between the conventional method of ocular prosthesis fabrication and a recent method using 3D printing technology on the patient satisfaction. Subjects and Methods: Twelve patients undergone enucleation of one eye were selected and randomly divided into two groups (6 patients of each). Group 1: each patient received the tradition ocular prosthesis. Group 2: each patient received the 3D printing ocular prosthesis. The patient satisfaction regarding the esthetics, comfort level, motility, socket secretion, weight and time of fabrication was evaluated after four weeks, then each patient was asked to wear the confirmer for one week. After that each patient of group1 received the 3D printing ocular prosthesis, and however each patient of group 2 received the conventional ocular prosthesis. The patient satisfaction was evaluated again after four weeks. Data were collected and subjected to analysis using Wilcoxon signed rank test. Results: There were statistical significance differences between 3D printing ocular prosthesis and conventional ocular prosthesis in the patient satisfaction regarding the esthetics, comfort level, motility, socket discharge, weight and time of fabrication. Conclusion: Using the 3D printing ocular prosthesis significantly increased the patients satisfaction more than conventional ocular prosthesis.
\end{abstract}

KEY WORDS: 3D ocular prosthesis, ocular prosthesis, patient satisfaction, 3D printing, Digital technology.

\section{INTRODUCTION}

In general, the eyes are the first facial features to be found that allow the human body to organize its tasks. The eyes are the components that interpret light, enabling the world to see how objects relate to each other, discern far from near objects, and decide their color and form. Eye removal not only induces unesthetic appearance, it also impacts, function and has a psychological effect on the patient, either due to tumors, trauma or some other illness ${ }^{(1)}$.
Eyeball could be removed due to one of the following causes; severe injury, cancer of the eye, congenital cystic eye, cosmetic improvement or constant infection in blind and disfigured eyes, panophthalmitis and in cases of sympathetic ophthalmia (inflammation of the eye) to prevent transmission to the other eye, which if left untreated can cause blindness. It can also be removed in a dead person, for their cornea to be used for a living person needing a corneal transplant by a surgical operation called keratoplasty or the need for histologic confirmation of a suspected diagnosis ${ }^{(2,3)}$.

1. Dentist, Kobry Al-Koba Military Hospital, Cairo, Egypt

2. Professor of Removable Prosthodontics Department, Faculty of Oral and Dental medicine, Al-Azhar University, Cairo, Egypt.

3. Professor and Chairman of Removable Prosthodontics Department, Faculty of Oral and Dental medicine, AlAzhar University, Cairo, Egypt.

-Corresponding author: dentist.hashem88@gmail.com 
Ocular prosthesis replaces an absent natural eye that fits under the eyelids. People with ocular prosthesis can do anything that normal people with both eyes are able to do, to the extent that others are not even aware that someone is wearing an artificial eye. The material used for fabricating an ocular prosthesis is mainly hard acrylic resin, for its numerous advantages ${ }^{(4,5)}$.

Two kinds of ocular prosthesis are available: (Readymade or custom- made): Readymade shells thatare readily available, cheapand can be assembledimmediately; and custommade prosthesis (CMP)that isto the actual person, and Personalized. CMP's got multiple benefits over stock shells,asbetterapposition to the socket's anterior side, better cosmesis and smoothereye movement. Although CMP is unquestionably a good choice for thepossibility that every patient needs ocular prosthesis, only trained ocularists will design it. Focus on the supply of eligible labor ${ }^{(6)}$.

The advantages of ocular prosthesis into achieving cosmetic purposes, filling the cavity left between the eyelids, prevention of the eye lashes from turning in and irritating the conjunctiva and also contributes to enhanced tissue health of the anophthalmic socket ${ }^{(7,8)}$.

A new breakthrough in the area of customised production of prostheses, Using innovative digital technology such as Computer Assisted Designing (CAD) and The potentialof Computer Assisted Manufacturing (CAM) Hold the key to an easy, inexpensive, and aesthetic key Ocular Prosthesis, technologically superior.

This sophisticated software assisted design synergy is technology and medical imaging andintegrates their distinctive characteristics with benefits, in terms of suit, to derive the best outcomes, convenience and beauty. This operation is referred to as Bio-CAD modelling and has proved to be extremely effective in facial, nasal, and auricular production Prothesis, prosthetics, and orthopedic replacements ${ }^{(9,10)}$.
Nowadays, CAD/3D printing systems was introduced in the market and used for fabrication of the artificial dental prosthesis, and it can be used for fabrication of the ocular prosthesis. However, till now there is a little study are available that compared between 3D printing ocular prosthesis and conventional ocular prosthesis. ${ }^{[10-12]}$ Therefore, this study was aimed to compare between the conventional method of ocular prosthesis fabrication and a recent method using 3D printing and face scanning technology. The null hypothesis of this study was that the difference in the patients satisfaction between the conventional ocular prosthesis and 3D ocular prosthesis would be insignificant.

\section{SUBJECTS AND METHODS}

Twelve patients were selected from out-patient clinic at the Department of Removable prosthodontic, Faculty of Dental Medicine, Al-Azhar university, to share in this study. The patients were clinically free from any systemic diseases, they have one eyeball completely enucleated, and they have large ocular bed. Ethical approval was obtained from the institutional ethical review committee EC Ref No.: 120192/3/24. Signed written consent forms were obtained from all patients before conducting any procedures. All selected patients were randomly equally divided and distributed into two groups (6 patients of each) as following: Group 1: each patient received the tradition ocular prosthesis. Group 2: each patient received the 3D printing ocular prosthesis.

\section{For each patient the following is carried on:}

- Extra-ocular examination was performed for the socket of the eye to detect any inflammation or secretion. Measurement of socket size and space available for eye prosthesis was performed, examination the muscles of the eye and movement of eyelids, and examination the old ocular prosthesis if present and patient complain examined..$^{(11)}$ 
- An accurate impression of the patient's eye socket is obtained to prepare a suitable wax model (Fig. 1).

- Position of the iris and the outer curvature are also determined on the wax model.

\section{For the conventional ocular prosthesis:}

- The wax model was flasked and processed into heat cured acrylic resin of the same color of the sclera of the other healthy eye of the patient, The iris then painted using oil pigments (Gouache oil, Northampton, USA) to the same color of the iris of another healthy eye of the patient, A layer of clear heat cured acrylic resin was added to shell and processed, Ocular prosthesis was deflasked and processed into heat cured PMMA (Scleral polymer white, USA) finished, polished, delivered to the patient, Fig. 2

\section{For the 3D printed ocular prosthesis:}

Computerized Tomography (CT) scan image by ( Toshiba Aquarium machine, Tokyo, Japanese) of the wax mold was made, The CT image is then converted into three-dimensional (3D) format using the Materialize Interactive Medical Image Control System (MIMICS) software, The 3D model that created was exported into a 3-Matic software which is an inbuilt module of MIMICS, The 3-Matic software refined the borders and contours and also creates a volume mesh, The model developed was fed into a rapid manufacturing machine which fabricated the prototype model of the ocular prosthesis (Fig.3), A face scanning was done $t$ for the patient face and healthy eye using highly accurate scanner (Bellus3D, Campbell, CA 95008, USA), MIMICS software was used for designing and constructed the ocular prosthesis from photopolymerised acrylic resin (PLA, Germany) and coloring it using the scanned image of the healthy iris with its colors to the prototype 3D model of the prosthetic eye on the predetermined position of the iris, (Fig,4).

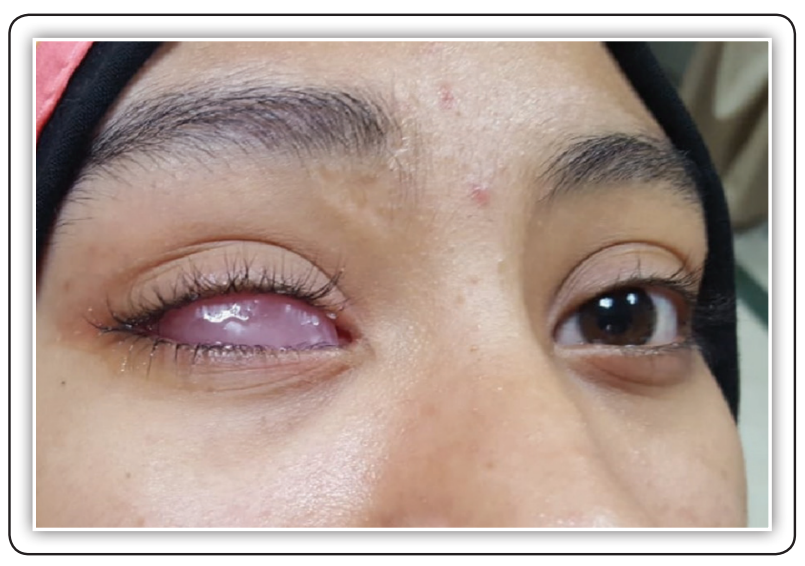

FIG (1) Suitable wax model try in

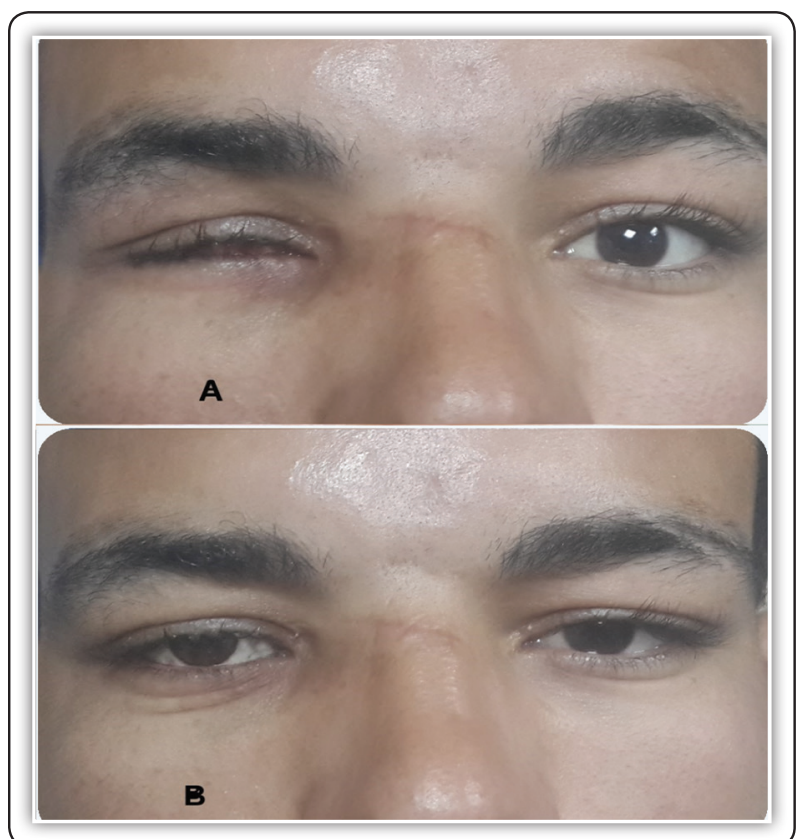

FIG (2) (A) Before and (B) after insertion of conventional ocular prosthesis in patient eye socket.

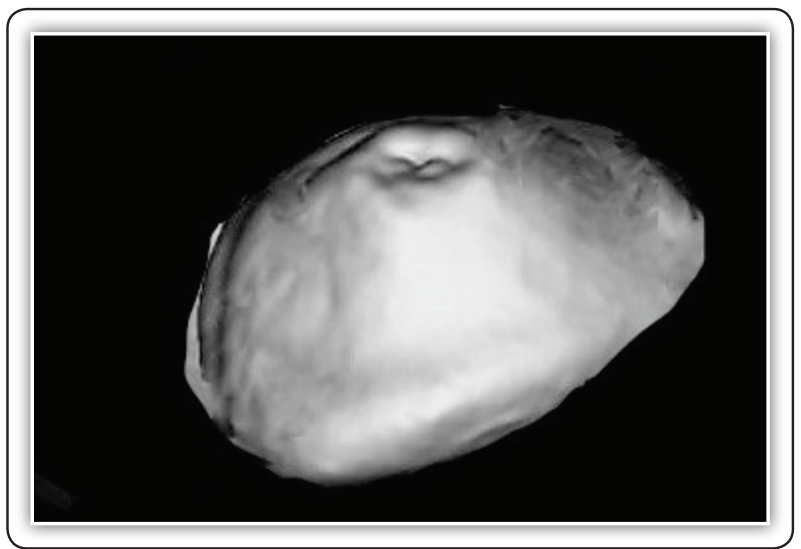

FIG (3) The prototype model of the ocular prosthesis 


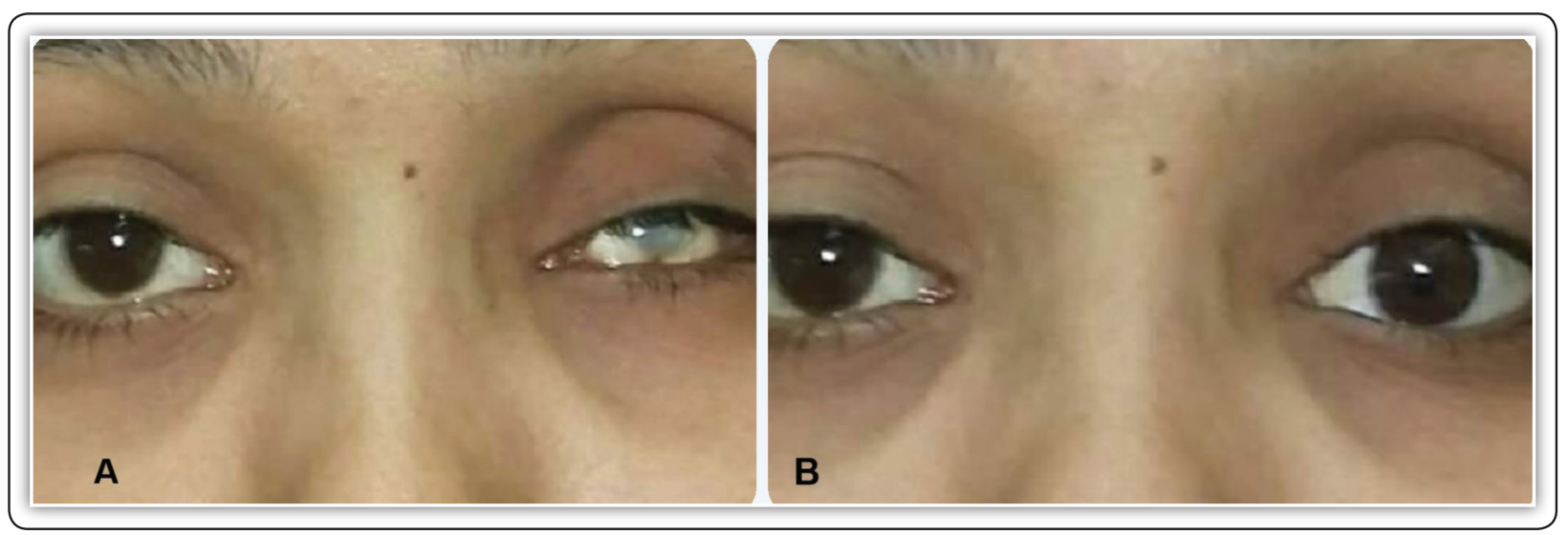

FIG (4) (A) Before and (B) after insertion of Rapid 3-D printing ocular prosthesis in patient eye socket.

Each patient of each group was asked to wear the ocular prosthesis for four weeks, after that the patient satisfaction was evaluated by asking the patient about his opinion regarding the esthetics, comfort level, motility, socket discharge, weight and time of fabrication.

Each item of the esthetics, comfort level, motility having 3 categories (bad, good and excellent), also socket discharge had 3 categories (mild, moderate and sever), while the weight item had 2 categories (heavy and light), also time of fabrication item had 2 categories (slow and fast).

However the scoring was carried out using the following scoring system; 1 represent as bad, slow, heavy, or sever, 2 represent as good, fast, light or moderate, and 3 represent as excellent or mild. ${ }^{(10,12)}$

Then that the patient was asked to wear his own conformer (that delivered to him after surgery) for one week.

- After that each patient in group (1) received 3D ocular prosthesis and each patient in group (2) received conventional ocular prosthesis.

- Each patient of each group was asked to wear the ocular prosthesis for four weeks, and the patient satisfaction was evaluated again by asking the patient about his opinion regarding the esthetics, comfort level, motility, socket discharge, weight and time of fabrication as mentioned above.

Data were presented as frequencies (n) and percentages (\%) and was analyzed using Wilcoxon signed rank test. The significance level was set at $\mathrm{p} \leq$ 0.05 for all tests. Statistical analysis was performed with IBM ${ }^{\circledR}$ SPSS ${ }^{\circledR}$ (SPSS Inc., IBM Corporation, NY, USA) Statistics Version 26 for Windows.

\section{RESULTS}

\section{1- Esthetics:}

Frequencies and percentage values for esthetics grading were presented in Table (1), 50.0\% of cases grading of esthetics for the conventional prosthesis as "Bad", however $75.0 \%$ of cases grading of esthetics for the 3D printed ocular prosthesis was reported as "Excellent". Statistically there was a significant difference between the grading of the two devices $(\mathrm{p}=0.001)$.

\section{2- Comfort:}

Frequencies and percentage values for comfort grading were presented in table (2), $66.70 \%$ of the cases grading of comfort for the conventional ocular prosthesis was reported as "Good"and $83.3 \%$ of the cases grading of comfort for the 3D printed ocular prosthesis was reported as "Excellent". Statistically there was a significant difference between the grading of the two devices ( $\mathrm{p}=0.004)$. 
TABLE (1) Frequencies and percentage values for esthetics grading.

\begin{tabular}{|c|c|c|c|c|c|}
\hline \multicolumn{3}{|c|}{ Parameter } & Conventional & 3D printed & p-value \\
\hline \multirow{6}{*}{ 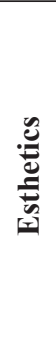 } & \multirow{2}{*}{ Bad } & $\mathbf{N}$ & 6 & 0 & \multirow{6}{*}{$0.001 *$} \\
\hline & & $\%$ & $50.0 \%$ & $0 \%$ & \\
\hline & \multirow{2}{*}{ Good } & $\mathbf{N}$ & 5 & 3 & \\
\hline & & $\%$ & $41.7 \%$ & $25.0 \%$ & \\
\hline & \multirow{2}{*}{ Excellent } & $\mathbf{N}$ & 1 & 9 & \\
\hline & & $\%$ & $8.3 \%$ & $75.0 \%$ & \\
\hline
\end{tabular}

TABLE (2) Frequencies and percentage values for comfort grading

\begin{tabular}{|c|c|c|c|c|c|}
\hline \multicolumn{3}{|c|}{ Parameter } & Conventional & 3D printed & p-value \\
\hline \multirow{6}{*}{ 苞 } & \multirow{2}{*}{ Bad } & $\mathbf{N}$ & 3 & 1 & \multirow{6}{*}{$0.004 *$} \\
\hline & & $\%$ & $25.0 \%$ & $8.3 \%$ & \\
\hline & \multirow{2}{*}{ Good } & $\mathbf{N}$ & 8 & 1 & \\
\hline & & $\%$ & $66.7 \%$ & $8.3 \%$ & \\
\hline & \multirow{2}{*}{ Excellent } & $\mathbf{N}$ & 1 & 10 & \\
\hline & & $\%$ & $8.3 \%$ & $83.3 \%$ & \\
\hline
\end{tabular}

\section{3- Motility:}

Frequencies and percentage values for motility gradingwere presented in table (3), 50.0\% of the cases grading of motility for the conventional ocular prosthesis was reported as "Bad" and $75.0 \%$ of the cases grading of motility for the 3D printed ocular prosthesis was reported as "Excellent". Statistically there was a significant difference between the grading of the two devices $(\mathrm{p}=0.002)$.

TABLE (3) Frequencies and percentage values for motility grading

\begin{tabular}{|c|c|c|c|c|c|}
\hline \multicolumn{3}{|c|}{ Parameter } & Conventional & 3D printed & p-value \\
\hline \multirow{6}{*}{ 胥 } & \multirow{2}{*}{ Bad } & n & 6 & 0 & \multirow{6}{*}{$0.002 *$} \\
\hline & & $\%$ & $50.0 \%$ & $0 \%$ & \\
\hline & \multirow{2}{*}{ Good } & n & 5 & 3 & \\
\hline & & $\%$ & $41.7 \%$ & $25.0 \%$ & \\
\hline & \multirow{2}{*}{ Excellent } & n & 1 & 9 & \\
\hline & & $\%$ & $8.3 \%$ & $75.0 \%$ & \\
\hline
\end{tabular}

\section{4- Level of socket discharge:}

Frequencies and percentage values for level of socket discharge were presented in table (4), 58.3\% of cases grading of level of socket discharge of the conventional ocular prosthesis was reported as moderate discharge while $58.3 \%$ of cases grading of level of socket discharge for the 3D printed ocular prosthesis was reported as a mild discharge during using prosthesis. There was a significant difference regarding the level of socket discharge of the two devices $(\mathrm{p}=0.025)$.

TABLE (4) Frequencies and percentage values for level of socket discharge

\begin{tabular}{|c|c|c|c|c|c|}
\hline \multicolumn{3}{|c|}{ Parameter } & Conventional & 3D printed & p-value \\
\hline \multirow{6}{*}{ 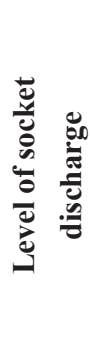 } & \multirow{2}{*}{ Mild } & n & 3 & 7 & \multirow{6}{*}{$0.025^{*}$} \\
\hline & & $\%$ & $25.0 \%$ & $58.3 \%$ & \\
\hline & \multirow{2}{*}{ Moderate } & $\mathbf{n}$ & 7 & 4 & \\
\hline & & $\%$ & $58.3 \%$ & $33.3 \%$ & \\
\hline & \multirow{2}{*}{ Severe } & $\mathbf{n}$ & 2 & 1 & \\
\hline & & $\%$ & $16.7 \%$ & $8.3 \%$ & \\
\hline
\end{tabular}

\section{5- Final weight:}

Frequencies and percentage values for prosthesis final weight were presented in table (5), $91.7 \%$ of cases grading of prosthesis final wight for the conventional ocular prostheses was reported as heavy prosthesis, while $66.7 \%$ of cases grading of prosthesis final wight for the 3D printed ocular prostheses was reported as light prosthesis. Statistically there was a significant difference regarding the final weight of the two devices $(\mathrm{p}=0.008)$.

\section{6- Time of fabrication:}

Frequencies and percentage values for time of fabrication were presented in table (6), $75.0 \%$ of cases grading for time of fabrication for the conventional ocular prostheses was reported as slow fabrication time, while $83.3 \%$ of cases grading for time of fabrication for the 3D printed ocular prostheses was reported as quickly fabricated time. Statistically 
there was a significant difference regarding the final weight of the two devices $(p=0.020)$.

TABLE (5) Frequencies and percentage values for prosthesis final weight

\begin{tabular}{|c|c|c|c|c|c|}
\hline \multicolumn{3}{|c|}{ Parameter } & Conventional & 3D printed & p-value \\
\hline \multirow{4}{*}{ 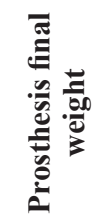 } & \multirow{2}{*}{ Heavy } & $\mathbf{n}$ & 11 & 4 & \multirow{4}{*}{$0.008 *$} \\
\hline & & $\%$ & $91.7 \%$ & $33.3 \%$ & \\
\hline & \multirow{2}{*}{ Light } & n & 1 & 8 & \\
\hline & & $\%$ & $8.3 \%$ & $66.7 \%$ & \\
\hline
\end{tabular}

TABLE (6) Frequencies and percentage values for time of fabrication

\begin{tabular}{|c|c|c|c|c|c|}
\hline \multicolumn{3}{|c|}{ Parameter } & Conventional & 3D printed & p-value \\
\hline \multirow{4}{*}{ 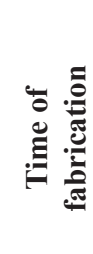 } & \multirow{2}{*}{ Slow } & $\mathbf{n}$ & 9 & 2 & \multirow{4}{*}{$0.020 *$} \\
\hline & & $\%$ & $75.0 \%$ & $16.7 \%$ & \\
\hline & \multirow{2}{*}{ Fast } & $\mathbf{n}$ & 3 & 10 & \\
\hline & & $\%$ & $25.0 \%$ & $83.3 \%$ & \\
\hline
\end{tabular}

\section{DISCUSSION}

Cross over longitudinal study was carried out on 12 patients to evaluate of ocular prosthesis fabricated by rapid 3-d printing technology versus ocular prosthesis constructed with conventional method.

Causes of eyeball removal were recorded to exclude the possibility of recurring infection or diseases in the future, depending on the severity of the situation, the surgical management may include one of 3 approaches: evisceration, enucleation, or exenteration, evisceration is the surgical procedure where the intraocular contents of the globe are removed, leaving the sclera, Tenon's capsule, conjunctiva, extra ocular muscles, and optic nerve undisturbed, the cornea may be retained or excised, each patient require at least 2-3 month after surgery to allow healing of socket.
Presence of an old prosthesis and the patient satisfaction regarding this prosthesis were recorded, also the patient's regular care and check up were included in the record, to avoid the future complaints and enhance the patient satisfaction for the newly constructed prosthesis.

Patient with old ocular prosthesis was asked to stop using it, until complete recovery of eye socket from any inflammation or secretions. The eye of intact side must be in healthy condition to give correct record to color of prosthetic eye and the size of sclera. The patients were medically free from any systemic diseases that may affect healing of the socket also Patients under radiation therapy were excluded from this study.

Patient with any socket deformation that may complicate fabrication of the prosthesis and the patients with inadequate ocular hygiene were excluded from this study due to continuous inflammation and socket discharge. Two custom made ocular prosthesis were made for all patients, one fabricated by acrylic resin as conventional method and the other made by Rapid 3-D Printing technology.

During ocular impression of ocular socket, the socket was anaesthetized, using local anesthesia (benoxinate eyedrops) to reduce the pain, the impression was taken by using highly flow material to allow injecting the impression material through ocular stock tray and to insure recording the fine details of the socket.

The impression was evaluated for proper extension and registration of the ocular socket, rubber base impression material was used to prepare a mold for the final impression due to it's easy manipulation and recording features, after complete setting of the rubber base mold, the mold was divided into two half, the mold was opened and the impression material and the tray were removed, to make a negative image, the molten inlay wax was poured through the hole made by the stem of the tray, during cooling, more molten wax was added 
to compensating any further shrinkage of the wax pattern, after complete cooling of the wax pattern, it was removed from the mold and all sharp edges and irregularities were smoothened and rounded to reduce the inflammation stimulation.

- In the conventual method, the wax pattern was processed into (heat-cured) grayish white or yellowish white acrylic resin to simulate the sclera color of intact eye ,Shell of a suitable scleral shade was made after deflasking by clear acrylic resin (heat-cured) to simulate the brightness of intact eye.

- The construction of 3-D printed ocular prosthesis, the wax pattern was scanning by a Computerized Tomography (CT) scan image by (Toshiba aquarium machine)to transfer it to 3D image by using [ Materialize Interactive Medical Image Control System (MIMICS) software ] then it was printed with proper color shade using 3D machine to fabricate the 3D prototype.

Each patient was asked to wear the conformer for one weak as rest period between two prostheses to prevent the effect of each type of prosthesis during evaluation of the patient satisfaction of another type.

The results of this study revealed that there was significant increase in patient satisfaction regarding the esthetics, comfort level, motility, socket discharge, weight and time of fabrication for the 3D printing ocular prosthesis more than that of conventional constructed ocular prosthesis. The pervious results were in accord with Alam et al. ${ }^{(10)}$ and Ko et al ${ }^{(12)}$.

The 3D prototype ocular prosthesis had significant increased the patient satisfaction regarding the esthetics more than conventional ocular prosthesis, $75 \%$ of cases grading of the Rapid 3-D Prototype ocular prosthesis was recorded as excellent, while $50 \%$ of cases grading of the conventional ocular prosthesis was recorded as bad, the 3D prototype ocular prosthesis had better esthetics with minimum error due to it is coloration was made by software.
The 3D prototype ocular prosthesis had significant increased the patient satisfaction regarding the comfort level more than conventional ocular prosthesis, $83.3 \%$ of cases grading of the Rapid 3-D Prototype ocular prosthesis was recorded as excellent $8.3 \%$ recorded as bad, while $8.3 \%$ of cases grading of the conventional ocular prosthesis was recorded as excellent and 25\% recorded as bad, this was due to $3 \mathrm{D}$ prototype ocular prosthesis having accurate size, minimum sharp edges and highly polished surface which reduce the inflammatory sign.

The $3 \mathrm{D}$ prototype ocular prosthesis had significant increased the patient satisfaction regarding the motility more than conventional ocular prosthesis, $75 \%$ of cases grading of the Rapid 3-D Prototype ocular prosthesiswas recorded as excellent, while $50 \%$ of cases grading of the conventional ocular prosthesis was recorded as bad motility, this due to the $3 \mathrm{D}$ prototype ocular prosthesis had proper size with minimum shrinkage and more adaptation to fitting surface of the socket.

The 3D prototype ocular prosthesis had significant increased the patient satisfaction regarding the level of socket discharge more than conventional ocular prosthesis, 58.3\% of cases grading of the Rapid 3-D Prototype ocular prosthesis was recorded as mild socket discharge, while $25 \%$ of cases grading of the conventional ocular prosthesis was recorded as mild socket discharge, this due to the 3D prototype ocular prosthesis had minimum polymerization cycle that decreased inflammation stimulation.

The 3D prototype ocular prosthesis had significant increased the patient satisfaction regarding the final weight of the prosthesis more than conventional ocular prosthesis, $66.7 \%$ of cases grading of the Rapid 3-D Prototype ocular prosthesis was recorded as light prosthesis, while $91.7 \%$ of cases grading the conventional ocular prosthesiswas recorded as heavy prosthesis, final weight of the $3 \mathrm{D}$ prototype ocular prosthesis was lighter in wight than conventional ocular prosthesis due to the difference in materials that used. 
The 3D prototype ocular prosthesis had significant increased the patient satisfaction regarding the time of fabrication of the prosthesis more than conventional ocular prosthesis, $83.3 \%$ of cases grading of the Rapid 3-D Prototype ocular prosthesis was recorded as fast fabrication prosthesis, while $75 \%$ of cases grading of the conventional ocular prosthesiswas recorded as slow fabrication prosthesis, this was due to $3 \mathrm{D}$ prototype ocular prosthesis having minimum steps and fasting printing machine, this was in accord with Ko et al. ${ }^{(12)}$.

This study recommends evaluating the using of new digital technology CAD/CAM and CAD/3D printing in construction of ocular prosthesis in large scale study.

\section{CONCLUSION}

Within the limitations of this study, the following conclusion was drawn:

Using the 3D printing ocular prosthesis significantly increased the patient satisfaction more than conventional ocular prosthesis.

\section{REFERENCES}

1. Marani E, Heida C. Eye and Orbit: The Eye Sees What You Do not Spot. Head and Neck: Springer; 2018. p. 221-57.

2. Perman KI, Baylis HI. Evisceration, enucleation, and exenteration. Otolaryngologic clinics of North America. 1988;21(1):171-82.

3. Kumar P, Aggrawal H, Singh RD, Chand P, Jurel SK, Alvi H, et al. A simplified approach for placing the iris disc on a custom made ocular prosthesis: Report of four cases. The Journal of Indian Prosthodontic Society. 2014;14(1):124-7.
4. Yom KH, Ko AC. History of Ocular Implants. Anophthalmia: Springer; 2020. p. 43-56.

5. Murphey PJ, Schlossberg L. Eye replacement by acrylic maxillofacial prosthesis. The Military Surgeon (United States). 1945;96(6):469-78.

6. Farook TH, Rahman AM, Nizami MMUI, Amin M, Jamayet NB, Alam MK. Custom made ocular prosthesis for acquired eye defect: Adefinitive option of rehabilitation. Bangladesh Journal of Medical Science. 2019;18(4): 823-6.

7. Shankaran G, Deogade SC, Dhirawani R. Fabrication of a cranial prosthesis combined with an ocular prosthesis using rapid prototyping: a case report. Journal of dentistry (Tehran, Iran). 2016;13(1):68.

8. Barman J, Bhattacherjee S, Rahman R. Fabrication of custom-made ocular prosthesis using conventional technique. International Journal of Oral Health Dentistry. 2020;6(1).

9. Farook T, Jamayet N, Abdullah J, Rajion Z, Alam M. A systematic review of the computerized tools and digital techniques applied to fabricate nasal, auricular, orbital and ocular prostheses for facial defect rehabilitation. Journal of stomatology, oral and maxillofacial surgery. 2020;121(3):268-77.

10. Alam MS, Sugavaneswaran M, Arumaikkannu G, Mukherjee B. An innovative method of ocular prosthesis fabrication by bio-CAD and rapid 3-D printing technology: A pilot study. Orbit. 2017;36(4):223-7.

11. Aggarwal H, Kumar P, Singh RD, Baslas V, Alvi HA, Jurel S. A multidisciplinary approach for ocular rehabilitation following surgical treatment of retinoblastoma: One year follow-up. Niger Med J. 2013;54(4):265-7.

12. Ko J, Kim SH, Baek SW, Chae MK, Yoon JS. Semi-automated fabrication of customized ocular prosthesis with three-dimensional printing and sublimation transfer printing technology. Scientific reports. 2019;9(1):1-8. 